\title{
A Novel Intra-Domain Continues Handover Solution for Inter-Domain Pmipv6 Based Vehicular Network
}

\author{
Haidar N. Hussain, Kamalrulnizam Abu Bakar \\ Faculty of Computer Science \& Information Systems \\ Universiti Teknologi Malaysia (UTM) \\ Johor, Malaysia
}

\author{
Shaharuddin Salleh \\ Faculty of Science Dept. Of Mathematics \\ Universiti Teknologi Malaysia (UTM) \\ Johor, Malaysia
}

\begin{abstract}
IP mobility management protocols (e.g. host based mobility protocols) incur significant handover latency, thus aggravate QoS for end user devices. Proxy Mobile IPv6 (PMIPv6) was proposed by the Internet Engineering Task Force (IETF) as a new network-based mobility protocol to reduce the host based handover latency. However the current PMIPv6 cannot support the vehicles high mobility while the vehicles motion within PMIPv6 domain. In this paper we introduce a novel intra-domain PMIPv6 handover technique based vehicular network using Media Independent Handover (MIH). The novel intra-domain PMIPv6 handover based vehicular network improves the handover performance of PMIPv6 by allowing the new PMIPv6 domain to obtain the MIIS information to estimate whether the handover is necessary or not before the vehicles movement to the second MAG of the new PMIPv6 domain. We evaluate the handover latency and data packet loss of the proposed handover process compared to PMIPv6. The conducted analysis results confirm that the novel handover process yields the reduced handover latency compared to that of PMIPv6 and also prevents data packet loss.
\end{abstract}

Keywords- PMIPv6; MIH; MIIS.

\section{INTRODUCTION}

The advanced wide deployment of wireless technologies in next generation networks laid the foundation stone of vehicular communication allowing vehicles to connect with the internet during movement. However, the vehicle will experience service interruption during the handover process, i.e., vehicle change its attachment point while maintaining ongoing sessions, due, to distinguishable handover latency and data packet loss.

Recently the developed PMIPv6 network-based protocol [5] allows an unaltered and mobility-unaware vehicle to change its attachment point while maintaining network connectivity. Compared with previous developed host-based mobility management protocols such as Mobile IPv6, Fast Mobile IPv6 (FMIPv6), and Hierarchical Mobile IPv6 (HMIPv6) [9] normally these host-based mobility protocols that are introduced by the Internet Engineering Task Force (IETF) for IP-based networks focus mainly on Mobile IP (MIP) [7]. IETF has developed both MIPv4 [6] and MIPv6. The importance of MIPv6 development is to support one of the most important requirements, which is the efficient support of mobility to provide continuous connectivity.

PMIPv6 is a desirable mobility management protocol designed for telecommunication service providers as well as manufacturers. As PMIPv6 is deployed in mobile networks, manufacturers do not need to implement a mobility stack for the vehicle. Form the telecommunication aspect, they can easily manage control the mobility services [12] Moreover, from the viewpoint performance, PMIPv6 generally outperforms the developed host-based mobility management protocols [8].

Accordingly, the investigation and analysis on the handover process of PMIPv6, which is expected to be a base mobility management protocol for next generation wireless networks (NGWN), is required. In addition, a proposal for improving the handover performance is a desirable work. Nevertheless, in recently published literatures [8] [14], improvements for the handover process of PMIPv6 have not been considered. In this paper, we introduce a novel intra-domain handover solution support for vehicles that cross the inter-domain PMIPv6 network (two different PMIPv6 networks) as far as our concern this is the first intra-domain PMIPv6 based vehicular ad-hoc networks research work done in PMIPv6 area.

The rest of this paper is organized as follows: section II introduces current PMIPv6 handover process. In section III we introduce the novel intra-domain handover solution design for solving the disconnection problem when roaming between two PMIPv6 domains and switching between to MAGs in the new LMA domain. In section IV we develop an analytical model to evaluate our novel intra-domain handover solution. Then, in section V, we present the conducted analysis result. Finally, section VI, concludes this paper.

\section{A. Proxy Mobile ipv6 (PMIPv6)}

The Internet Engineering Task Force designed Proxy Mobile IPv6 (PMIPv6) to support network-based IP mobility management for MNs, without requiring its involvement in any related IP-mobility functions. Mobility management in PMIPv6 is provided to $\mathrm{MN}$ irrespective of the presence or absence of Mobile IPv6 functionality [6]. Fig 1 shows the conceptual PMIPv6 domain.

PMIPv6 extends the signaling of MIPv6 and reuses most of MIPv6 concepts such as HA functionality. In addition it introduces two new elements known as Local Mobility Anchor (LMA) and Mobile Access Gateway (MAG) [4]. The LMA behaves similar to the HA in MIPv6 in the PMIPv6 domain and also it introduces additional capabilities required for networkbased mobility management [2].

PMIPv6 supports the MN within a topological localized domain by utilizing the MAG entity. MAG organizes with the 
access routers and handles mobility signaling on behalf of the $\mathrm{MN}$.

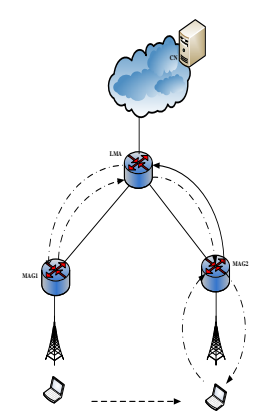

Figure 1. PMIPv6 network architecture

PMIPv6 protocol operation consists of four phases. In the first phase, MAG retrieves the MN's profile using its current identifier. The Binding Update (BU) is the second phase, in which the MAG sends a Proxy Binding Update (PUB) request to the LMA in order to register the current point of attachment of the MN. Accordingly, a binding cache entry and a tunnel for the MN's home prefix will be created. The third phase will be the MAG emulating the mobile node's home interface on the access interface. Therefore, the MN will always believe it is in the home network. Fourthly, the LMA replies with a Proxy Bind Acknowledge (PBA) message with the MN's HNP. After receiving the Router Advertise (RA) message, the MN configures its IP address by using the contained prefix. For packet routing, the LMA is able to route all received packets over the established tunnel to the MAG. The MAG forwards these packets to the MN. Additionally, the MAG will relay all the received packets over the tunnel to the LMA and then they will be routed towards the CN. Fig 2 shows the procedure when a MN joins a PMIPv6 domain.

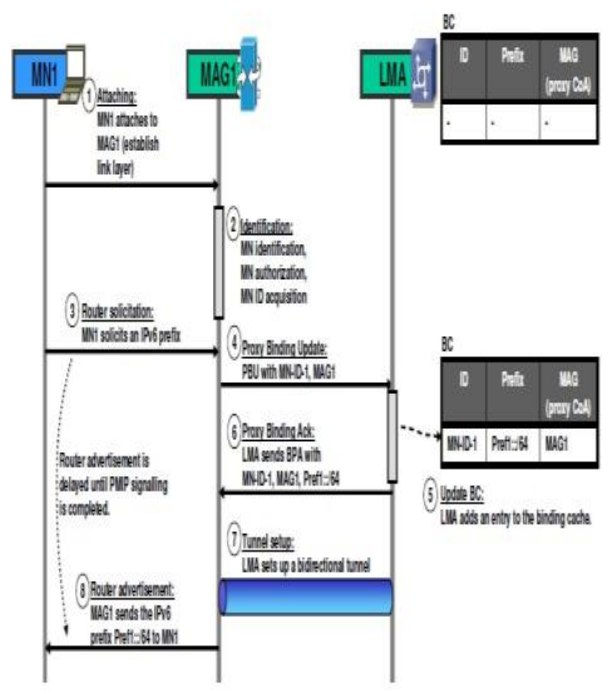

Figure 2. the procedure of a MN joining a PMIPv6 domain.

While the MN is roaming in the PMIPv6 domain, the protocol ensures that the $\mathrm{MN}$ is eligible to obtain its home address on any access link [2] on condition that it is roaming in the same PMIPv6 domain. That is, that the serving PMIPv6 assigns a unique home network prefix, Pre-MN-Prefix, to each $\mathrm{MN}$ and this prefix conceptually follows the MN were ever it moves within the PMIPv6 domain [3].

As a result there is no need to perform address configuration to reconfigure a new address for the MN every time it changes its point of attachment. This in turn, optimizes handover performance by reducing the latency that is caused because of the address configuration. Also, because of the MAG network element which performs the network signaling on behalf of the MN, PMIPv6 reduces the binding update delay by reducing the round trip time, thus reducing handover latency. The procedure of the attachment is explained as follows:

a) Attaching:

MN1 attaches to the MAG1 through a point-to-point link and establishes the link layer. Any access technology is possible provided that it emulates a point-to-point behavior (e.g. PPP, PPPoE).

b) Identification:

MAG1 authenticates MN1 based on its link layer address (e.g. MAC address) and ascertains what MN1 is permitted to do (authorization). The authorization step may use existing services like LDAP or RADIUS.

c) Router solicitation:

MN1 sends a router solicitation to obtain an IPv6 prefix. MAG1 will not send a router advertisement until it obtained a prefix for MN1 from the LMA (step 6, PBA).

d) Proxy binding update (PBU):

MAG1 sends a proxy binding update to the LMA. This PBU associates the MAG1 address with the identity of the MN.

e) Allocate prefix, update $\mathrm{BC}$ :

The LMA allocates a prefix for MN1 (Home Network Prefix). The LMA creates an entry in its BC. The entry contains the MN1 ID (MN-ID-1), the address MAG1 of the proxy MAG (proxy-CoA) as well as the prefix assigned to $\mathrm{MN} 1$.

f) Proxy binding ACK (PBA):

The LMA sends a PBA back to MAG1. The PBA contains the information of the BPC entry created in step 5.

g) Tunnel setup:

The LMA and MAG1 establish a bidirectional IPv6in-IPv6 tunnel that is used for tunneling packets to and from MN1. The LMA sets a route through the tunnel for traffic that is addressed to the MN.

\section{B. Media Independent Handover IEEE 802.21}

Media Independent Handover (MIH) specification is primitive to provide link layer information and other related information to the upper layers to optimize handover. Fig 3 shows MIH architecture. One of the most important categories is the Event Service (MIES); it is used for the Layer 3 handover hints. Events provide the data link layer conditions to layer 3 or 
reflect the response of Layer 3. The representative event primitives include link going down, link down, and link going up. Layer 3 handover occurs when the vehicle changes its point of attachment after an inter-domain movement (inter-network or intra-foreign-network-movement). Inter-domain movement means that the vehicle changes its attachment point between two different networks. When layer 3 handover occurs, the vehicle will lose its IP-connectivity; it hence will lose the ongoing transmission. In addition MIH provides Command Services (MICS); it includes a set of commands that might be sent from higher layers to lower layers, and Information Services (MIIS); provides a set of information, including query/response structure to allow vehicles to discover and obtain information about available networks in a geographical area, attachment capabilities/ network point of attachment, and other information related to the network.

MIH provides these services to MIH user Service Access Point (SAP). MIH-SAP and MIH-Link-SAP serve as MIHF interfaces to L3 and above layers, in addition to lower layers (L2 and below) respectively.

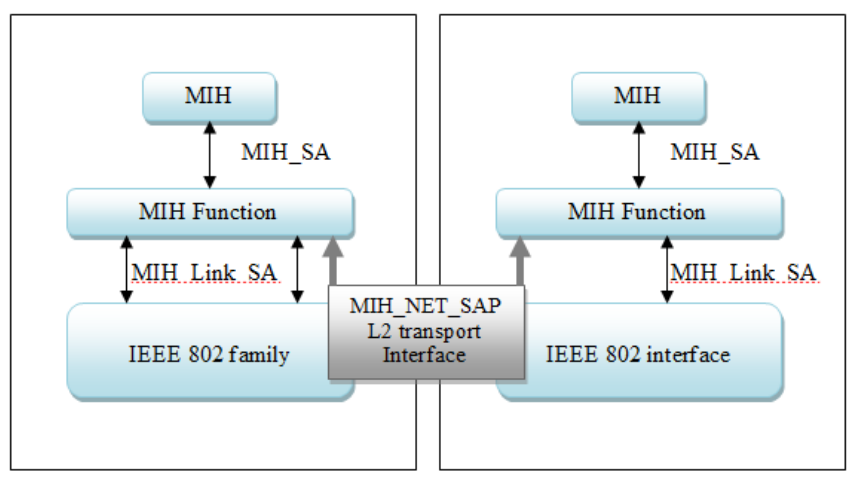

Figure 3.

MIH architecture

\section{NOVEL INTRA-DOMAIN PMIPV6 HANDOVER TECHNIQUE}

We extend the proposed inter-domain PMIPv6 handover scheme in [1] to support intra-domain PMIPv6 by defining new MIH primitives and parameters. As shown in table 1 and Fig 4 below; a new primitive is introduced in our proposed handover scheme known as "MIH-Prefix Info" (Media Independent Handover Prefix-Information).

The MIH-Prefix Info will contain information about the current serving PMIPv6 network domain. The stored information pulled by MIHF of the serving MAG represents lower and upper layer (e.g. L2, L3) information of the serving PMIPv6 domain. MAG pulls the information from the vehicle using MIH-links list and MIH-link Available. In this novel intra-PMIPv6 handover technique we suggest that the "Prefix" parameter is added to these primitives and thereby the serving MAG gets the information by pulling and using the prefix. Fig 5 shows the proposed handover mechanism using MIH.

We propose a Homogeneous Network Information (HNI) Container and it is assumed that the HNI is embedded within the vehicle, Logical interface and is connected with a PMIPv6 domain using MIH (IEEE 802.21) services PMIPv6 architecture that is shown in Fig 4.
TABLE I. NEW PROPOSED PRIMITIVES AND PARAMETERS

\begin{tabular}{|l|c|ll|}
\hline \multicolumn{1}{|c|}{ Primitives } & Service & \multicolumn{1}{|c|}{ Parameters } \\
\hline MIH-PrefixInfo & CS & Interface ID, Prefix & \\
\hline MIH-Link List & IS & $\begin{array}{l}\text { Interface ID, Prefix, MAC } \\
\text { Address, BW, Quality Level }\end{array}$ \\
\hline MIH-LinkAvailable & ES & $\begin{array}{l}\text { Interface ID, Prefix, MAC } \\
\text { Address, BW, Quality Level }\end{array}$ \\
\hline MIH-LinkGoingDown & ES & $\begin{array}{l}\text { Interface ID, MAC Address, BW, } \\
\text { Quality Level }\end{array}$ \\
\hline MIH-LinkDown & ES & $\begin{array}{l}\text { Interface ID, Prefix, MAC } \\
\text { Address }\end{array}$ \\
\hline MIH-LinkUp & ES & $\begin{array}{l}\text { Interface ID, Prefix, MAC } \\
\text { Address }\end{array}$ \\
\hline
\end{tabular}

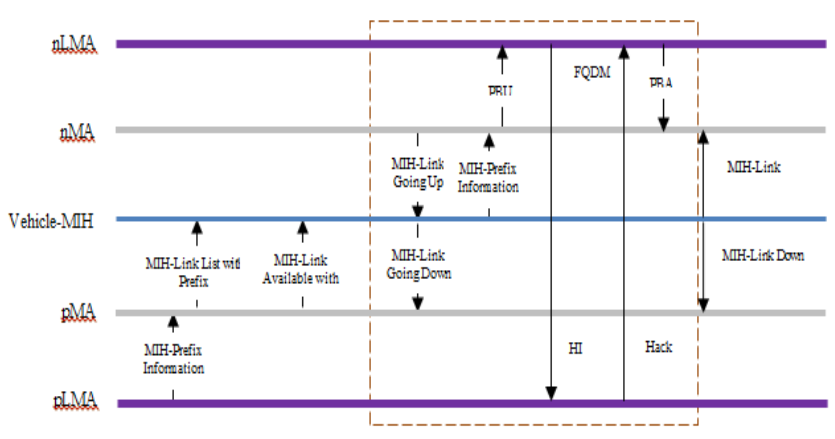

Figure 4. proposed handover mechanism using MIH

HNI defined to facilitate the storage and information retrieval of the L2 and L3 static information of current and neighboring networks obtained through the IEEE 802.21 MIIS. The IE known as the 'MIH-PrefixInfo' is used to provide prefixes information about the current network and neighboring networks. Alongside with L2 information, they form the proposed pre-defined (HNI) container. Hence, the information will be used by the LMA once it receives information about the vehicles link statues based on this information the LMA will generate a bi-directional tunnel with the next new MAG (i.e. MAG 2 in the new domain) as shown in Fig 6. In addition we propose a logical layer embedded within layer 3 to help support reducing the handover latency by allowing the vehicle to connect with MAG (i.e. MAG2) seamlessly while maintaining it active connection with the first nMAG (i.e. MAG1).

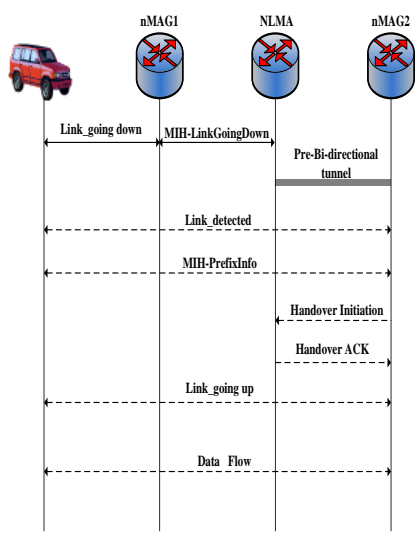

Figure 5. novel intra-domain PMIPv6 handover signaling

We summarize the process of our proposed intra domain scheme as listed below: 
- When the serving MAG senses that the vehicle is about to disconnect a link going down event, it will inform the LMA of this event. MAG is enabled to trigger the link event using MIH-Link going down.

- LMA by receiving this indication from the serving MAG will pre-establish a tunnel with the next serving MAG (we assume that the vehicle moves in one direction).

- Once the next MAG (nMAG2) senses the vehicles signal within the overlap area it will pull HNI container and will send it to the LMA. Then nMAG2 will emulate the vehicles to its connection link by establishing the connection through the logical interface.

- The serving LMA will remove the tunnel with the pMAG and the packets will be forwarded through the new path.

By using the logical interface LMA will not buffer the packets until the handover with the next nMAG is completed but it will directly send the packets to the vehicle through the logical interface.

\section{ANALYTICAL MODEL AND ANALYSIS}

\section{A. Network Model}

Fig 6 shows the network model, which includes vehicle, $\mathrm{BS}$, mobile access gateway (MAG), local mobile anchor (LMA), and correspondent node (CN). There are two LMA domains and each LMA has n MAGs. The coverage of LMA is called domain, and the coverage of BS is known as cell. In other words, each domain has $n$ cells. A BS connected to a MAG has a wireless interface for connecting vehicle (s). In this paper, we suppose that the vehicle has moved for inter-domain PMIPv6 to intra-domain PMIPv6 (in this case the process will be an intra-domain process whereby the vehicle is roaming within the same LMA domain).

Furthermore, we assume that once the vehicle passes the overlap area the nLMA will be able to intelligently calculate the stay time of the vehicle within the communication range of the first serving MAG based on the MIH information.

We adopt the vehicle mobility model in where the direction of the vehicle motion in an LMA domain is uniformly distributed on $[0,2 \pi]$.

For simplicity, we assume that the shape of the coverage area of a MAG is circular (non-circular areas, such as hexagonal shaped areas, can be reasonably approximated with the same size) and an intra-PMIPv6 consists of nMAGs with the same size of the coverage area of $\mathrm{aS}_{\mathrm{AR}}$. Vehicle (s) move at an average velocity of $\mathrm{V}$.

Let $\mu_{c}, \mu_{d}$ be cell crossing rate and domain crossing rate, respectively. Furthermore, let $\mu_{\mathrm{I}}$ be the cell crossing rate for that vehicle which is within the same PMIPv6 domain.

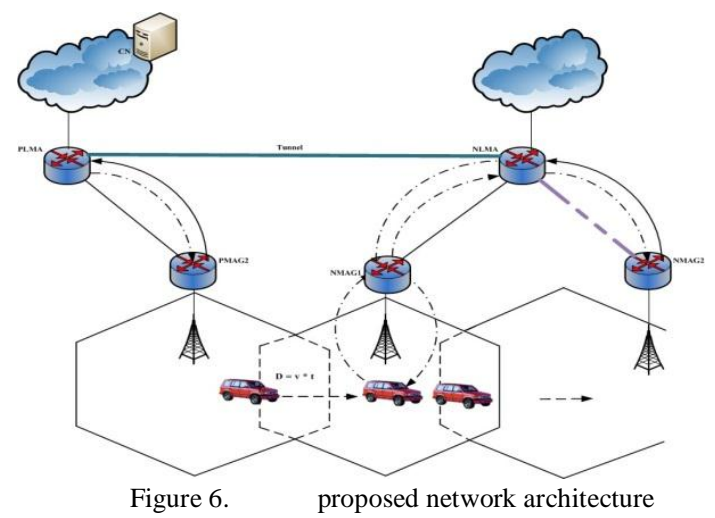

Assuming that each AR has a coverage area of $S_{A R}$, the border crossing is given by [13].

$$
\begin{aligned}
& \mu_{c}=\frac{2 v}{\sqrt{\pi S_{A R}}} \\
& \mu_{d}=\frac{2 v}{\sqrt{\pi n S_{A R}}}=\frac{\mu_{c}}{\sqrt{n}} \\
& \mu_{I}=\mu_{c}-\mu_{d}=\frac{\sqrt{n-1}}{\sqrt{n}} \mu_{c}
\end{aligned}
$$

The residence time in a cell and in a domain follows exponential distribution with parameters $\mu_{c}$ and $\mu_{d}$, while session arrival process follows a Poisson distribution with rate $\lambda_{s}$. Hence, the average number of cell crossing and domain crossing can be obtained as follows:

$$
\begin{aligned}
& E\left(N_{c}\right)=\frac{\mu_{c}}{\lambda_{s}}, \\
& E\left(N_{d}\right)=\frac{\mu_{d}}{\lambda_{S}} .
\end{aligned}
$$

From (2), and (5), we obtain:

$E\left(N_{d}\right)=\frac{1}{\sqrt{n}} \frac{\mu_{c}}{\lambda_{S}}$

Let $\mathrm{E}\left(\mathrm{N}_{\mathrm{I}}\right)$ be the average number of cell crossing rate of a vehicle, which is in the same PMIPv6 domain (intra-domain). The expression will be as follows

$E\left(N_{I}\right)=\frac{\mu_{I}}{\lambda_{S}}$

Crossing between to LMA domains, a vehicle crosses between two subnets. Thus, from (4) and (5), the average number of intra-domain handover $\mathrm{E}\left(\mathrm{N}_{\mathrm{s}}\right)$ is given by (8).

$$
E\left(N_{s}\right)=E\left(N_{c}\right)-E\left(N_{d}\right)=\frac{\mu_{c}}{\lambda_{S}}-\frac{1}{\sqrt{n}} \frac{\mu_{c}}{\lambda_{S}}=\frac{\mu_{c}}{\lambda_{S}}\left(1-\frac{1}{\sqrt{n}}\right)
$$


For a system that follows a Poisson Process, the delay experienced by packets that are transmitted within the network will be equaled to the transmission delay and the propagation time delay at every link in the network. The network links are combination of both wired and wireless links with the failure probability of $\mathrm{P}_{\mathrm{f}}$.

\section{B. Perameter Analysis}

We analyze the important performance metrics such as handover latency $L_{H}^{(.)}$which is defined as the time interval during the vehicle cannot receive any data packets while performing the handover process. The notations used for our analysis are as introduced in [9] as listed in table 2:

TABLE II. TABLE 2 THE NOTATIONS USED IN THE ANALYTICAL MODELING

\begin{tabular}{|c|c|}
\hline $\mathrm{T}_{\mathrm{L} 2}$ & $\begin{array}{l}\text { Link-layer handover latency, which mainly depends } \\
\text { on an implementation chipset for a wireless interface }\end{array}$ \\
\hline $\mathrm{T}_{\mathrm{WRS}}$ & $\begin{array}{l}\text { The random amount of delay before sending an } \\
\text { initial RS message }\end{array}$ \\
\hline $\mathrm{T}_{\mathrm{RS}}$ & $\begin{array}{l}\text { The arrival delay of the RS message sent from the } \\
\text { MN to the MAG at the new access network }\end{array}$ \\
\hline $\mathrm{T}_{\mathrm{LU}}^{(\mathrm{PMIPv} 6)}$ & $\begin{array}{l}\text { The delay of the location update (registration) for } \\
\text { the MN }\end{array}$ \\
\hline $\mathrm{T}_{\mathrm{P}}^{(\mathrm{PMIPv} 6)}$ & $\begin{array}{l}\text { The arrival delay of the first packet sent from the } \\
\text { LMA to the MN }\end{array}$ \\
\hline $\mathrm{T}_{\mathrm{P}}^{(\mathrm{PRO})}$ & $\begin{array}{l}\text { is the arrival delay of the first packet sent from the } \\
\text { MAG to the MN }\end{array}$ \\
\hline $\mathrm{T}_{\mathrm{BS}}$ & The buffering start time \\
\hline $\mathrm{T}_{\mathrm{BE}}$ & The buffering end time \\
\hline $\mathrm{T}_{\mathrm{L} 2-\mathrm{REP}}^{\text {(PRO) }}$ & $\begin{array}{l}\text { The arrival delay of the L2 report sent from the MN } \\
\text { to the MAG }\end{array}$ \\
\hline $\mathrm{T}_{\mathrm{DREG}}^{(\mathrm{PRO})}$ & The delay of the de-registration for the MN \\
\hline $\mathrm{T}_{\mathrm{HNPP}}^{(\mathrm{PRO})}$ & $\begin{array}{l}\text { The delay of the HNPP (Home network Prefix } \\
\text { process) }\end{array}$ \\
\hline $\mathrm{M}_{\mathrm{S}}^{(\mathrm{X})}$ & $\begin{array}{l}\text { The size of the message } X \text {, which is used in the } \\
\text { handover i.e., } X \in\{\mathrm{PBU}, \mathrm{RS}, \mathrm{HD}, \mathrm{DATA}\}\end{array}$ \\
\hline
\end{tabular}

\section{1) Handover latency of PMIPv6}

Despite the handover timing diagram of PMIPv6 handover process shown in Fig 7. Let $\mathrm{L}_{\mathrm{H}}^{\text {(PMIPv6) }}$ represents the handover latency of PMIPv6 handover process. Then, $\mathrm{L}_{\mathrm{H}}^{(\mathrm{PMIPv} 6)}$ will be expressed as:

$\mathrm{L}_{\mathrm{H}}^{(\text {PMIPv6) }}=\mathrm{T}_{\mathrm{L} 2}+\mathrm{T}_{\mathrm{WRS}}+\mathrm{T}_{\mathrm{RS}}+\mathrm{T}_{\mathrm{LU}}^{(\text {PMIPv6 })}+\mathrm{T}_{\mathrm{P}}^{(\text {PMIPv6) }}$

\section{Where}

$T_{W R S}$ is determined as a value between 0 and MAX_RTR_SOLICITATION $N_{D E L A Y}$ (Narten \& T., Nordmark, E., \& Simpson W., 1998). It is assumed that $T_{W R S}$ is uniformly distributed in the interval $[0$, MAX_RTR_SOLICITATION_DEELAY].

$T_{R S}$ is calculated as:

$$
\begin{gathered}
\mathrm{T}_{\mathrm{RS}}= \\
\left(\frac{\mathrm{M}_{\mathrm{S}}^{(\mathrm{RS})}}{\mathrm{b}_{\mathrm{WL}}}+\mathrm{t}_{\mathrm{WL}}\right)+\left(\frac{\mathrm{M}_{\mathrm{S}}^{(\mathrm{RS})}}{\mathrm{b}_{\mathrm{WL}}}+\mathrm{t}_{\mathrm{WL}}\right) * \\
\sum_{\mathrm{n}_{\mathrm{f}}}^{\infty} \mathrm{n}_{\mathrm{f}} * \operatorname{Prob}\left\{\mathrm{n}_{\mathrm{f}} \text { failures and 1 success }\right\},
\end{gathered}
$$

Where $M_{S}^{(R S)}$ is the RS message size. $b_{W L}$ is the bandwidth of the wireless link between MN and MAG. $t_{W L}$ is the wireless link propagation time. $n_{f}$ is the number of message failures over the wireless link. Suppose $P_{f}$ is the link failure probability. By applying $P_{f}$ in equation (10), it is rewritten as [10]:

$$
\mathrm{T}_{\mathrm{RS}}=\left(\frac{\mathrm{M}_{\mathrm{S}}^{(\mathrm{RS})}}{\mathrm{b}_{\mathrm{WL}}}+\mathrm{t}_{\mathrm{WL}}\right)+\left(\frac{\mathrm{M}_{\mathrm{S}}^{(\mathrm{RS})}}{\mathrm{b}_{\mathrm{WL}}}+\mathrm{t}_{\mathrm{WL}}\right) * \frac{\mathrm{P}_{\mathrm{f}}}{1-\mathrm{P}_{\mathrm{f}}}
$$

Figure 7. PMIPv6 Handover timing diagram

It is further assumed that the wired link between LMA and MAG is robust and no message failure is expected. Then $T_{L U}^{(P M I P v 6)}$ in equation 1 is obtained as:

$$
\mathrm{T}_{\mathrm{LU}}^{(\mathrm{PMIPv6})}=\mathrm{n}_{\mathrm{h}}\left(\frac{\mathrm{M}_{\mathrm{S}}^{(\mathrm{PBU})}}{\mathrm{b}_{\mathrm{WD}}}+\mathrm{t}_{\mathrm{WD}}\right),
$$

Where $M_{S}^{(P B U)}$ is the size of the PBU message. $n_{h}$ is the number of link hopes between the MAG and LMA. $b_{W D}$ is the bandwidth of the wired link between the MAG and LMA. $t_{W D}$ is the is the propagation time for the wired link. And, $T_{P}^{(P M I P v 6)}$ is obtained by:

$$
\begin{aligned}
\mathrm{T}_{\mathrm{P}}^{(\text {PMIPv6) }}=\mathrm{n}_{\mathrm{h}} & \left(\frac{\mathrm{M}_{\mathrm{S}}^{(\mathrm{HD})}+\mathrm{M}_{\mathrm{S}}^{(\mathrm{DATA})}}{\mathrm{b}_{\mathrm{WD}}}+\mathrm{t}_{\mathrm{WD}}\right)+2\left(\frac{\mathrm{M}_{\mathrm{S}}^{(\mathrm{DATA})}}{\mathrm{b}_{\mathrm{WL}}}+\right. \\
& \left.\mathrm{t}_{\mathrm{WL}}\right) * \frac{\mathrm{P}_{\mathrm{f}}}{1-\mathrm{P}_{\mathrm{f}}} \quad(14)
\end{aligned}
$$

Where $M_{S}^{(H D)}$ and $M_{S}^{(D A T A)}$ are the sizes of IPv6 header and messages, respectively. Recall data packets for the $\mathrm{MN}$ are traversed via the bi-directional tunnel established between the LMA and new MAG (i.e. MAG2). In addition the LMA knows about the MN attachment with the new MAG as it receives the new MAG PBU message.

\section{2) Novel Intra-domain PMIPv6 solution}

Depicts the handover timing diagram of the intra-domain PMIPv6 handover technique the handover process is shown in Fig 8.

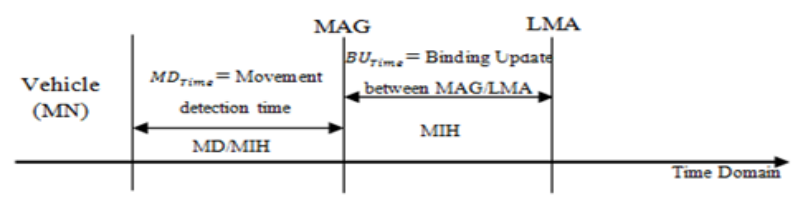

Figure 8. Handover latency time domains of the proposed Interdomain PMIPv6 
Let $L_{H}^{(P \text { intra-domainPMIPv6) }}$ be the handover latency for the proposed intra-domain PMIPv6 handover latency. Then, it is expressed as:

$$
\mathrm{L}_{\mathrm{H}}^{\text {(intra-domain PMIPv6 Solution) }}=\mathrm{T}_{\mathrm{L} 2}+2 * \mathrm{~T}_{\text {vehicle,MAG }}
$$

Furthermore, the processing time for the novel intra-domain PMIPv6 is calculated as shown below:

$$
\mathrm{T}_{\mathrm{L} 2}+2 * \mathrm{~T}_{\mathrm{vehicle}, \mathrm{MAG}}+\mathrm{n}_{\mathrm{h}}\left(\frac{\mathrm{M}_{\mathrm{S}}^{(\mathrm{PBU})}}{\mathrm{b}_{\mathrm{WL}}}+\mathrm{t}_{\mathrm{WL}}\right)
$$

In equation (16), the data transmission delay over the wired link between LMA and the MAG is excluded, which has taken account in the basic handover process as shown in equation (14). This, is because the proposed intra-domain PMIPv6 handover process allows the nLMA to establishes a bidirectional tunnel with the next nMAG1 that falls in the vehicle movement direction; while the vehicle is within the overlap area of the first new nMAG1 and second nMAG2 within the nLMA. Then the LMA forwards the packets destined to the vehicle through the next nMAG2. Accordingly, as soon as the vehicle attaches to the $\mathrm{MAG}$, it will receive the data packets through this MAGs BS.

For the comparison purpose, we define the relative gain of handover latency to the basic handover process as:

$$
\mathrm{G}_{\mathrm{H}}^{(.)}=\frac{\mathrm{L}_{\mathrm{H}}^{(.)}}{\mathrm{L}_{\mathrm{H}}^{\text {(proposed) }}}
$$

Where, $\mathrm{G}_{\mathrm{H}}^{(.)}$is used for indicating a relative handover performance gain compared with other handover schemes, $\mathrm{L}_{\mathrm{H}}^{(.)}$ represents the handover latency for an existing handover scheme, and $\mathrm{L}_{\mathrm{H}}^{\text {(proposed) }}$ represents the proposed handover scheme. For instance, if $\mathrm{G}_{\mathrm{H}}^{\text {(intra-domain) }}$ is larger than 1.0 , it means that the proposed handover process outperforms the basic one.

Hence by applying equation 17 and by assuming that layer 2 time equals to $400 \mathrm{~ms}$, the Gain will be:

$$
\begin{aligned}
& G_{\mathrm{H}}^{\text {(intra-domain) }}=\frac{\mathrm{L}_{\mathrm{H}}^{(\text {PMIPv6) }}}{\mathrm{L}_{\mathrm{H}}^{\text {(intra-domain })}} \\
& \mathrm{G}_{\mathrm{H}}^{\text {(intra-domain) }}=\frac{470}{391.5}=1.2
\end{aligned}
$$

By applying equation (17), we can notes that the gain of our novel intra-domain PMIPv6 handover technique is larger than 1.0. Hence our proposed technique outperforms the conventional PMIPv6 scheme.

\section{System ANALYSIS Result}

In this section, we use the parameters listed in Table 3 [1] [9] to calculate the handover latency for our proposed solution and compare it with PMIPv6 method. Figure 9 shows the handover latency for the novel intra-domain PMIPv6. We can notice that, the proposed intra-domain PMIPv6 solution can reduce the handover latency time compared with PMIPv6. Furthermore more fig 9 shows the impact of speed on the handover process our proposed novel intra-domain PMIPv6 technique outperformed the conventional PMIPv6 scheme in reducing the overall handover processing time in vehicular network environment. Furthermore Fig 10 shows the effect of the wireless link latency impact over the handover process the results show that the novel intra-domain PMIPv6 preforms better than PMIPv6. Hence, the proposed inter-domain PMIPv6

\begin{tabular}{|c|c|c|}
\hline \multirow{19}{*}{ Delay } & Notation & $\begin{array}{c}\text { Default } \\
\text { Value }\end{array}$ \\
\hline & $\mathrm{T}_{\mathrm{AAA}}$ & $30 \mathrm{~ms}$ \\
\hline & $\mathrm{T}_{\mathrm{PBU}}$ & $30 \mathrm{~ms}$ \\
\hline & $\mathrm{T}_{\mathrm{L} 2}$ & $200-400 \mathrm{~ms}$ \\
\hline & $\mathrm{T}_{\text {inter }}$ & $50 \mathrm{~ms}$ \\
\hline & $\mathrm{T}_{\mathrm{RA}}$ & 60 \\
\hline & $\mathrm{T}_{\mathrm{DAD}}$ & $\begin{array}{l}500-1000 \\
\mathrm{~ms}\end{array}$ \\
\hline & $\mathrm{T}_{\text {vehicle,MAG }}$ & $10 \mathrm{~ms}$ \\
\hline & $\mathrm{b}_{\mathrm{WD}}$ & $100 \mathrm{Mbps}$ \\
\hline & $b_{W L}$ & $11 \mathrm{Mbps}$ \\
\hline & $t_{W D}$ & $0.5 \mathrm{~ms}$ \\
\hline & $t_{W L}$ & $2 \mathrm{~ms}$ \\
\hline & $\mathrm{p}_{\mathrm{f}}$ & {$[0.1,0.4]$} \\
\hline & $\mathrm{n}_{\mathrm{h}}$ & 5 \\
\hline & $\mathrm{M}_{\mathrm{S}}^{\mathrm{RS}}$ & 52 byte \\
\hline & $\mathrm{M}_{\mathrm{S}}^{\mathrm{PBU}}$ & 76 bytes \\
\hline & $M_{S}^{(H D)}$ & 40 bytes \\
\hline & $\mathrm{M}_{\mathrm{S}}^{\mathrm{DATA}}$ & 120 bytes \\
\hline & $\lambda_{\mathrm{s}}$ & {$[0.3,0.7]$} \\
\hline
\end{tabular}
handover process outperforms the basic handover process of PMIPv6 in terms of handover latency and data packet loss.

TABLE III. TABLE 3 PARAMETERS TO CALCULATE THE PERFORMANCE METRICS

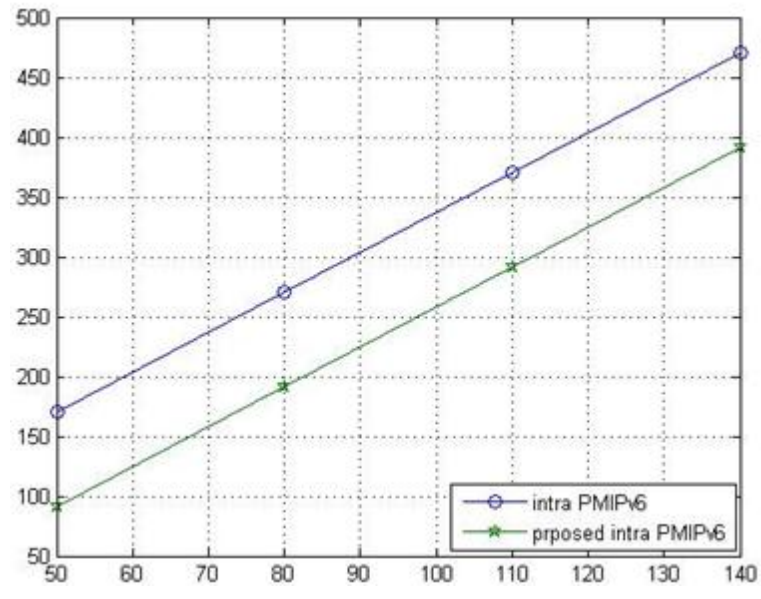

Figure 9. Handover latency vs. Vehicle Speed

\section{CONCLUSION}

In this paper, we proposed an intra-domain PMIPv6 handover technique for vehicular environment and compared our proposed technique with PMIPv6. 


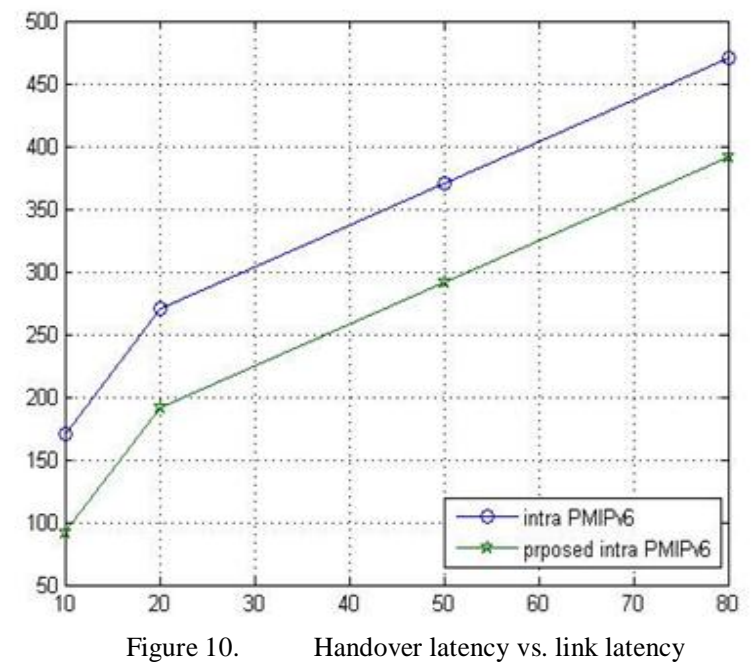

The proposed intra-PMIPv6 handover technique is based on MIIS information function.Using the MIH services (MIIS), the vehicle can obtain information without rout discovery or RtSolPr/PrRtAd messages. Thus, the handover latency time due to concurrent start of L2 and L3 handover process is reduced. In this way, our scheme is suitable for a cost-effective network compared with PMIPv6 scheme.

The novel intra-domain handover technique is proposed to solve the high mobility of the vehicle while moving from interdomain to intra-domain by using a logical interface to support continuance communication within the new PMIPv6 domain after completing the inter-domain PMIPv6 handover process.

Our future direction consists of plans to develop a mathematical model to evaluate the novel intra-domain PMIPv6 technique in different environments for both interdomain and intra-domain schemes. In addition we will propose a new handover estimation technique. Further investigation of the novel intra-domain technique will be tested on a highway vehicular scenario. The impact of the intra-domain and interdomain scheme on seamless connection support for vehicles roaming in PMIPv6 domains will be analytically analyzed.

\section{ACKNOWLEDGMENT}

The authors would like to thank to the administration of Faculty of Computer Science \& Information Systems and Universti Teknologi Malaysia for their encouragement and generous support.

\section{REFERENCES}

[1] AL-Hashimi, H. N., Kamalrulnizam Abu Bakar, \& Kayhan Zrar Ghafoor (2010). Inter-domain Proxy Mobile IPv6 based Vehicular Network.
Network Protocols and Algorithms .

[2] Arnold, T., W. Lloyd, J. Zhao, \& G. Cao. (2008). IP address passing for VANETs. IEEE Percom (pp. 70-79). IEEE.

[3] Bechler, M., \& L. Wolf. (2005). Mobility management for vehicular ad hoc networks. IEEE Vehicular Technology (pp. 2294-2298). IEEE.

[4] Fazio, M., C., P. E., S. , D., \& M. , G. (2007). Facilitating real-time applications in VANETs through fast address auto-configuration. IEEE, 981-985.

[5] Gundavelli, S., K. Leung, V. Devarapalli, K. Chowdhury, \& B. Patil. (2008, August). Proxy Mobile IPv6. Retrieved from IETF RFC 5213: http://www.ietf.org/rfc/rfc5213.txt

[6] Huang, C. M., M. S. Chiang, \& T. H. Hsu. (2008). PFC: A packet forwarding control scheme for vehicle handover over the ITS networks. Computer Communications, 2815-2826.

[7] Johnson, D., C., P., \& J, A. (2004). Mobility Support in IPv6. Retrieved fromhttp://www.ietf.org/rfc/rfc3775.txt: http://www.ietf.org/rfc/rfc3775.txt

[8] Lee, J.-H., \& Tai-Myoung Chung. (2010). Cost Analysis of IP Mobility Management Protocols for Consumer Mobile Devices. IEEE Consumer Electronics Society . IEEE.

[9] Lee, J.-H., Zhiwei Yan, \& Ilsun You. (2011). Enhancing QoS of Mobile Devices by a New Handover. Springer Science+Business Media.

[10] McNair, J., Ian F. Akyildizr, \& Michael 13. Bender. (2000). An intersystem handoff technique for. IEEE Annual Joint Conference of the IEEE Computer and Communications Societies (INFOCOM) (pp. 208216). IEEE.

[11] Narten, \& T., Nordmark, E., \& Simpson W. (1998, December). Neighbor discovery for IP version 6. Retrieved from In IETF RFC 2461.

[12] Sangheon Pack, Ilsun You, \& Tai-Myoung Chung. (2009). Enabling a paging mechanism in network-based. Journal of Internet Technology.

[13] XIAO, L., \& Jiawei YANG. (2010, 08 20). Performance analysis of proxy mobile IPv6 based on IEEE802.16e.

[14] Yan, Z., Huachun Zhou, \& Ilsun You. (2010). N-NEMO: A comprehensive network mobility solution in proxy mobile IPv6 network. ournal of Wireless Mobile Networks, Ubiquitous Computing, and Dependable Applications, 52-70.

\section{AUTHORS PROFILE}

Haidar N. AL-Hashimi is currently a $\mathrm{PhD}$ candidate studying in the Department of Computer Network \& Communication, Universiti Teknologi Malaysia, Johor, Malaysia. Haidar received his B.Sc degree in Computer Science and M.Sc degree in Computer Science/Unicast Routing in Mobile Ad hoc Networks in 2005 and 2009 respectively. His current research interests include Vehicular Ad Hoc Networks, Routing over VANET, and Handover Process in Heterogeneous Vehicular Networks.

Kamalrulnizam Abu Bakar obtained his $\mathrm{PhD}$ degree from Aston University (Birmingham, UK) in 2004. Currently, he is associate professor in Computer Science at Universiti Teknologi Malaysia (Malaysia) and member of the "Pervasive Computing" research group. He involves in several research projects and is the referee for many scientific journals and conferences. His specialization includes mobile and wireless computing, information security and grid computing.

Shaharuddin Salleh obtained his $\mathrm{PhD}$ degree from Universiti Teknologi Malaysia, Johor, Malaysia in Computational Mathematics (UTM) Currently, he is professor in Mathematic Department at Universiti Teknologi Malaysia (Malaysia). He involves in several research projects and is the referee for many scientific journals and conferences. His specialization Computational Mathematics \& Network modeling. 